\title{
Corticosteróides tópicos no tratamento da fimose primária em idade pediátrica: revisão baseada na evidência
}

Paulo Pires, * Hermínia Teixeira,* Irene Lopes,** José Agostinho Santos*

\section{RESUMO}

Objectivos: A fimose, definida como a presença de prepúcio não retráctil por anel prepucial estreito, pode classificar-se em primária ou secundária. A fimose primária está presente em $96 \%$ dos recém-nascidos e tende à regressão espontânea até aos 3 anos. A partir desta idade pondera-se intervenção terapêutica. A circuncisão é o tratamento clássico mas está associada a morbilidade e custos não desprezíveis. Recentemente, vários autores reportaram o uso de corticosteróides tópicos como tratamento alternativo com bons resultados. Assim, o objectivo deste trabalho foi rever a evidência disponível sobre a eficácia e a segurança da aplicação tópica de corticosteróides no tratamento da fimose primária dos 3 aos 18 anos.

Fontes de dados: Medline, sítios de medicina baseada na evidência, Índex de Revistas Médicas Portuguesas e referências bibliográficas dos artigos seleccionados.

Métodos de revisão: Pesquisa de normas de orientação clínica (NOC), revisões sistemáticas, meta-análises, ensaios clínicos aleatorizados e controlados (ECAC), utilizando as palavras-chave: phimosis e steroids. Foi utilizada a escala Strenght of Recommendation Taxonomy (SORT) da American Family Physician para classificar os artigos.

Resultados: Foram encontrados 44 artigos, dos quais quatro cumpriam os critérios de inclusão: uma NOC, que propõe o tratamento com corticosteróides tópicos $0,05 \%$ a $0,1 \%$, duas vezes por dia, durante 20 a 30 dias (SOR A); e três ECAC, nos quais se verificou que o uso de corticosteróides tópicos no tratamento da fimose primária é eficaz, com resolução em $65,8 \%$ a $90 \%$ dos casos, sem efeitos laterais descritos (nível de evidência 1).

Conclusões: A evidência disponível indica que o uso de corticosteróides tópicos no tratamento da fimose primária em idade pediátrica é eficaz e desprovido de efeitos laterais (SOR A). A aplicação do fármaco pressupõe a realização de manobras de retracção do prepúcio, que devem ser repetidas durante a higiene. Assim, constitui uma abordagem terapêutica ao alcance do Médico de Família.

Palavras-Chave: Fimose; Corticosteróides.

\section{INTRODUÇÃO}

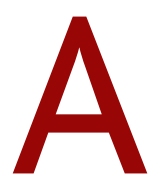

fimose define-se como a presença de um prepúcio não retráctil devido a uma abertura prepucial estreita ${ }^{1,2} \mathrm{e}$, segundo as características macroscópicas do anel prepucial, é classificada em primária ou secundária. Na primária não existem sinais de cicatrização. Na secundária, pelo

*Internos de Medicina Geral e Familiar, Centro de Saúde da Senhora da Hora. **Interna de Medicina Geral e Familiar, Unidade de Saúde Familiar Oceanos, Centro de Saúde de Matosinhos. contrário, são visíveis alterações cicatriciais, que ocorrem maioritariamente após contextos inflamatórios locais, como na balanite xerótica obliterante, podendo surgir num prepúcio previamente retráctil. ${ }^{1}$

Os termos fimose fisiológica e fimose patológica, apesar de frequentes na literatura, são utilizados com diferentes significados, pelo que não existe uma definição clara destes conceitos. ${ }^{1-4}$

A fimose primária afecta cerca de $96 \%$ dos recém-nascidos. Uma vez que apresenta um declínio notório com a idade, assiste-se a uma redução da prevalência 
para cerca de 10 a $20 \%$ aos 3 anos. $^{3-7}$ Diversos autores, com base no trabalho de Oster et al, salientam que, a partir dessa idade, uma maior percentagem de crianças apresentará uma resolução mais lenta da fimose até à adolescência, que pode mesmo não resolver e manter-se na idade adulta. Portanto, pondera-se o início de terapêutica a partir dos 3 anos de idade. ${ }^{3-6}$

A fimose primária pode ser sintomática quando associada a balanite, infecção do trato urinário (para as quais predispõe) ou ao início da actividade sexual. Além disso, está descrito um maior risco de carcinoma espinocelular peniano e de infecção pelo vírus da imunodeficiência humana em relações sexuais de risco. ${ }^{1,7,8}$

Kikiros, Beasley e Woodward estabeleceram uma classificação da fimose de acordo com o grau de retractilidade do prepúcio (Quadro I). Pacientes com graduação $\geq 3$ beneficiam de tratamento. ${ }^{2}$

A terapêutica clássica para a fimose é a circuncisão, tendo sido desenvolvida posteriormente a prepucioplastia. ${ }^{1,3}$ Uma vez que ambas são procedimentos cirúrgicos, têm custos mais elevados e estão associados a morbilidade não desprezível. ${ }^{1,2}$ São relativamente frequentes a infecção e a hemorragia incisionais e, mais raramente, a estenose e a fístula do meato uretral. Pode ainda surgir fimose secundária. O risco de complicações anestésicas, a ansiedade dos pais e os possíveis sintomas psíquicos consequentes à mutilação genital são outros aspectos relevantes inerentes à cirurgia., ${ }^{2,7,9}$

Nos últimos anos, vários investigadores reportaram o uso de corticosteróides tópicos como tratamento alternativo à cirurgia, com elevada taxa de sucesso e me-

\section{QUADRO I. Graduação da retractilidade do prepúcio} por Kikiros, Beasley e Woodward²

0 retracção total ou apenas limitada por aderências balano-prepuciais congénitas

1 retracção incompleta com anel constritivo no sulco coronal

2 retracção parcial com exposição da glande até à sua parte média

3 retracção parcial apenas com meato visível

4 retracção ligeira sem exposição do meato e da glande

5 sem retracção lhor relação custo/benefício. ${ }^{3,7}$ Supõe-se que, a nível biomolecular, o efeito anti-inflamatório e anti-fibrinolítico do fármaco resulte numa inibição da síntese de glicosaminoglicanos e assim favoreça uma diminuição da espessura da derme, o que permite gradualmente uma maior abertura prepucial.,

Assim, o objectivo dos autores é rever a evidência disponível sobre a eficácia e os efeitos laterais da aplicação tópica de corticosteróides no tratamento da fimose primária em indivíduos do sexo masculino com idade $\geq 3$ anos $\mathrm{e}<18$ anos.

\section{MÉTODOS}

Foi realizada uma pesquisa de normas de orientação clínica (NOC), revisões sistemáticas, meta-análises e ensaios clínicos aleatorizados e controlados (ECAC) na Medline, sítios de medicina baseada na evidência, Índex de Revistas Médicas Portuguesas e referências bibliográficas dos artigos seleccionados, publicados entre Janeiro de 2000 e Junho de 2010, nas línguas portuguesa, inglesa e espanhola, utilizando as palavras-chave (termos MeSH): phimosis e steroids.

Os artigos seleccionados incluíram indivíduos do sexo masculino com idade $\geq 3$ anos e $<18$ anos com fimose primária sem complicações, cuja intervenção fosse a aplicação tópica de corticosteróides no anel prepucial comparativamente a manobras de retracção do prepúcio com ou sem aplicação tópica de placebo. Os resultados avaliados foram a eficácia (taxa de resolução) e a segurança (efeitos laterais).

Foi utilizada a escala Strenght of Recommendation Taxonomy (SORT) da American Family Physician, ${ }^{10}$ para avaliação dos estudos e atribuição dos níveis de evidência e forças de recomendação.

\section{RESULTADOS}

Foram encontrados 44 artigos, dos quais quatro cumpriram os critérios de inclusão: uma NOC e três ECAC. Os restantes foram excluídos por divergirem do objectivo do trabalho, por não cumprirem os critérios de inclusão ou por serem repetidos.

A NOC, da autoria conjunta da European Association of Urology e da European Society for Paedriatric Urology, é baseada em evidência clínica, tendo sido publicada em Março de 2008 e revista, sem alterações, em Março de 2009. Para a terapêutica da fimose pri- 
mária estas duas sociedades propõem uma abordagem cirúrgica (circuncisão ou prepucioplastia) ou médica (corticosteróides tópicos). Tendo como base estudos de nível de evidência 1 , a aplicação tópica de corticosteróide $0,05 \%$ a $0,1 \%$, duas vezes por dia, durante 20 a 30 dias, é descrita como eficaz e desprovida de efeitos laterais, sendo proposta com uma força de recomendação A.

Os três ECAC seleccionados (Quadro II) concluíram que existe maior eficácia (entre 65,8\% e 90\%), sem efeitos laterais associados (potencialmente hemorragia $\mathrm{e}$ infecção locais, atrofia da pele, síndrome de Cushingiatrogénico, entre outros) com o uso de corticosteróides tópicos durante quatro semanas, comparativamente à realização de manobras de retracção do prepúcio com ou sem creme placebo.

Todos os ECAC utilizaram a escala de graduação da retractilidade prepucial proposta por Kikiros, Beasleye Woodward $^{2}$ (Quadro I), tendo incluído nos estudos crianças com grau de retractilidade $\geq 3$.

No estudo de $E s$ posito et al, ${ }^{3}$ que incluiu 240 meninos, com idades compreendidas entre os 3 e os 13 anos, com fimose graus 3 a 5 , o uso de furoato de mometasona a $0,1 \%$, duas vezes por dia, durante quatro semanas, associado a manobras de retracção do prepúcio durante a aplicação do fármaco e à higiene perineal, mostrou ser significativamente mais eficaz do que o placebo e sem efeitos laterais.

Zampieri et al utilizaram uma amostra de 298 meninos, entre os 4 e os 14 anos, com fimose grau 5 e distribuíram-na homogeneamente por três grupos terapêuticos. O grupo A foi submetido a aplicação bi-diária de betametasona a 0,05\%, durante 15 dias, seguida da aplicação única diária durante mais 15 dias. No grupo B foi utilizada a mesma terapêutica mas com aplicação única diária do corticosteróide durante 30 dias. Em ambos os grupos, os pais foram aconselhados a realizar manobras de retracção do prepúcio durante a aplicação do creme, bem como durante a limpeza genital. No grupo $\mathrm{C}$ foram usadas apenas manobras de retracção do prepúcio. Verificou-se uma maior resolução da fimose nos grupos A e B, submetidos à aplicação de corticosteróide associada a manobras, sendo superior no grupo A, submetido ao tratamento inicial bi-diário (90\% versus $72 \%$, respectivamente). Não foram descritos efeitos laterais em nenhum dos grupos.

Um outro ECAC, de Lund et al, ${ }^{6}$ realizado na China, avaliou 137 meninos entre os 3 e os 15 anos, com fimose

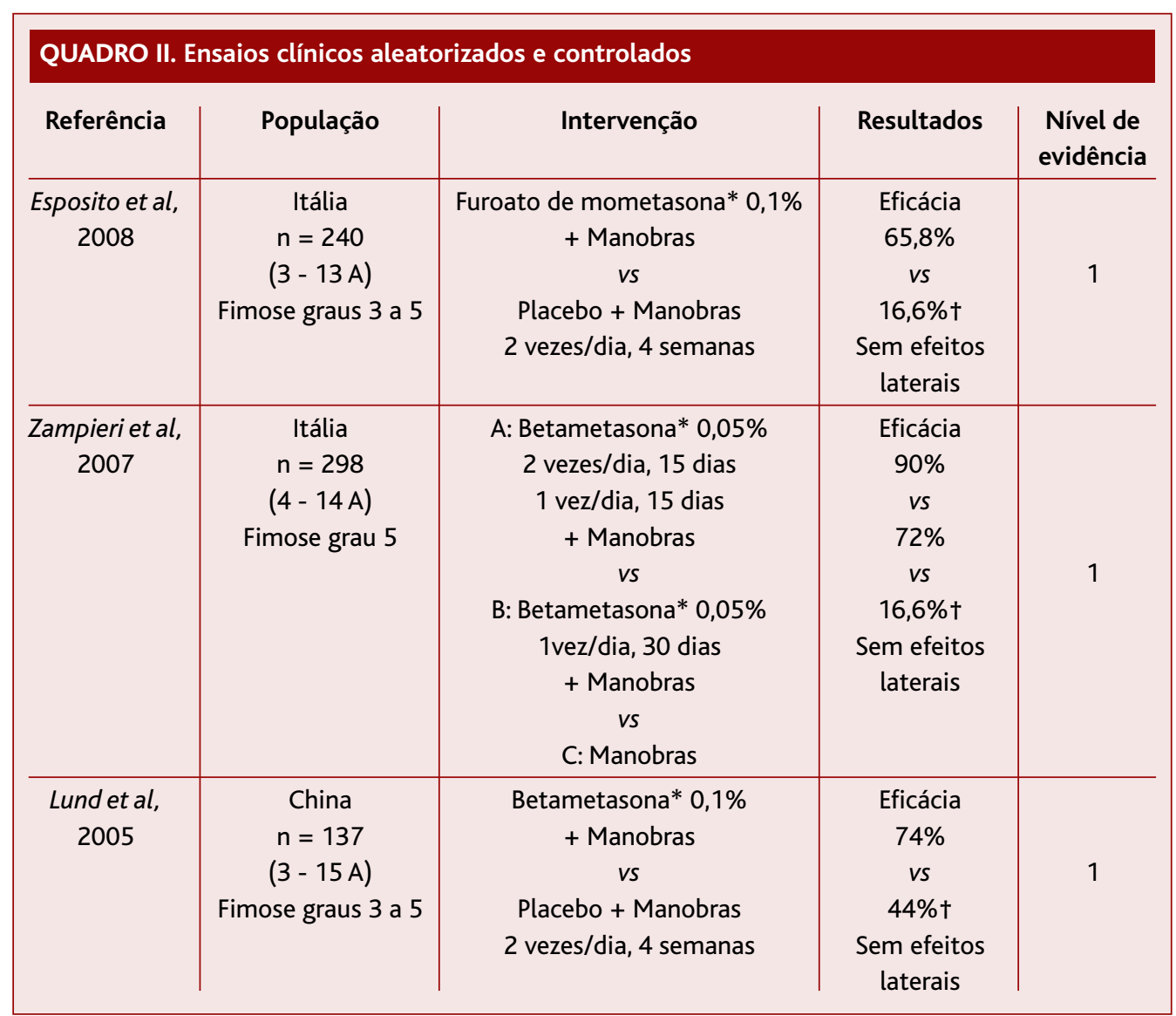

* Corticosteróides de elevada potência; † Diferença estatisticamente significativa. 
graus 3 a 5. Registou uma eficácia significativamente superior na resolução da fimose com o uso de betametasona a $0,1 \%$, duas vezes por dia, durante quatro semanas, associada a manobras de retracção prepucial comparativamente ao uso de placebo com manobras $(74 \%$ versus $44 \%$ respectivamente). Neste estudo também não foram descritos efeitos laterais.

Os três ECAC apresentam boa amostragem, são duplamente cegos com atribuição oculta, têm um seguimento superior a $80 \%$ e um resultado orientado para o paciente, pelo que, os autores desta revisão lhes atribuíram nível de evidência 1.

\section{CONCLUSÕES}

A evidência disponível sobre a eficácia e segurança da aplicação tópica de corticosteróides no tratamento da fimose primária é, assim, muito consistente. Com base na NOC e nos ECAC analisados, os autores recomendam o uso de corticosteróide tópico $0,05 \%$ a $0,1 \%$, especificamente mometasona e betametasona, até duas vezes por dia, num ciclo de quatro semanas, para o tratamento da fimose primária sem complicações associadas, em crianças com idades compreendidas entre os 3 e os 15 anos (força de recomendação A).

Na bibliografia é realçada a importância da capacitação e colaboração dos pais. Uma aplicação correcta do corticosteróide tópico pressupõe uma manobra de retracção do prepúcio delicada e indolor, após a qual uma camada fina de fármaco é colocada no anel prepucial. $^{3-6}$

Em todos os ECAC seleccionados o início do tratamento foi precedido por uma explicação aos pais sobre a aplicação do fármaco e a avaliação das crianças ocorreu logo após quatro semanas de tratamento. Assim, os autores desta revisão sugerem que seja tomada uma conduta semelhante sempre que se opta por esta terapêutica. A manutenção das manobras de retracção do prepúcio durante a higiene genital é importante para prevenção de recidiva. Para tal, a colaboração dos pais é fundamental. ${ }^{3,5,9} \mathrm{O}$ número de ciclos de quatro semanas a implementar mantém-se controverso. Alguns autores propõem um máximo de três ciclos para resolução da fimose primária antes da referenciação para intervenção cirúrgica. ${ }^{3,5}$

Assim, o uso de corticosteróides tópicos na fimose primária constitui uma abordagem terapêutica ao al- cance do Médico de Família e permitirá uma redução na referenciação para Urologia ou Cirurgia Pediátrica. Além disso, representa uma alternativa à abordagem cirúrgica que apresenta menores custos e riscos.

Tratando-se de uma opção terapêutica relativamente recente, algumas questões permanecem. São necessários mais estudos que incluam outros corticosteróides tópicos (nomeadamente de potências inferiores), que os comparem e que estabeleçam o número de ciclos de aplicação tópica a implementar antes da referenciação.

\section{REFERÊNCIAS BIBLIOGRÁFICAS}

1. Phimosis. In: Tekgul S, Riedmiller H, Gerharz E, Hoebeke P, Kocvara R, Nijman R, et al. Guidelines on Paediatric Urology. Arnhem: European Association of Urology, European Society for Paediatric Urology; 2009. p. 6-8.

2. Pileggi FO, Vicente YA. Phimotic ring topical corticoid cream $(0,1 \%$ mometasone furoate) treatment in children. J Pediatr Surg 2007 Oct; 42 (10): 1749-52

3. Esposito C, Centonze A, Alicchio F, Savanelli A, Settimi A. Topical steroid application versus circumcision in pediatric patients with phimosis: a prospective randomized placebo controlled clinical trial. World J Urol 2008 Apr; 26 (2): 187-90.

4. Ashfield JE, Nickel KR, Siemens DR, MacNeily AE, Nickel JC. Treatment of phimosis with topical steroids in 194 children. J Urol 2003 Mar; 169 (3): 1106-8

5. Zampieri N, Corroppolo M, Zuin V, Bianchi S, Camoglio FS. Phimosis and topical steroids: new clinical findings. Pediatr Surg Int 2007 Apr; 23 (4): 331-5.

6. Lund L, Wai KH, Mui M, Yeung CK. An 18-month follow-up study after randomized treatment of phimosis in boys with topical steroid versus placebo. Scand J Urol Nephrol 2005; 39 (1): 78-81.

7. Marques TC, Sampaio FJ, Favorito LA. Treatment of phimosis with topical steroids and foreskin anatomy. Int Braz J Urol 2005 Jul-Aug; 31 (4): 370-4.

8. Yang SS, Tsai YC, Wu CC, Liu SP, Wang CC. Highly potent and moderately potent topical steroids are effective in treating phimosis: a propective randomized study. J Urol 2005 Apr; 173 (4): 1361-3.

9. Lee JW, Cho SJ, Park EA, Lee SJ. Topical hydrocortisone and physiotherapy for nonretractile physiologic phimosis in infants. Pediatr Nephrol 2006 Aug; 21 (8): 1127-30.

10. Ebell MH, Siwek J, Weiss BD, Woolf SH, Susman J, Ewingman B, et al. Strength of Recommendation Taxonomy (SORT): a patient-centered approach to grading evidence in the medical literature. Am Fam Phys 2004 Feb 1; 69 (3): 548-56.

\section{AGRADECIMENTOS}

Aos Orientadores de Formação dos autores: Dra. Mónica Granja, Dra. Maria Cristina Rodrigues, Dra. Raquel Braga e Dra. Joana Santos, pela colaboração na elaboração deste trabalho. 
ENDEREÇO PARA CORRESPONDÊNCIA

Paulo Pires

Avenida da República, n. ${ }^{\circ}$ 354, 6. D $^{\circ}$ to Frt

4450 - 237 Matosinhos
E-mail: psrpires@gmail.com

Recebido em 22/11/2010

Aceite para publicação em 05/05/2011

\section{ABSTRACT}

\section{TOPICAL STEROIDS IN THE TREATMENT OF PRIMARY PHIMOSIS IN CHILDREN: AN EVIDENCE-BASED REVIEW}

Goals: Phimosis is defined as the presence of a non-retractable foreskin by a narrow preputial ring. It can be classified as primary or secondary. Primary phimosis is present in $96 \%$ of newborns, and tends to resolve spontaneously in the first 3 years of life. After this age treatment may be considered. Circumcision is the classic treatment, but is associated with morbidity and costs. Recently, several authors have reported the use of topical steroids as an alternative treatment for phimosis with good results. The aim of this review is to evaluate the available evidence on the efficacy and safety of topical steroids in the treatment of primary phimosis in children aged 3 to 18 years.

Data sources: Medline, evidence-based medicine web sites, the Index of Portuguese Medical Journals and references of selected articles.

Review methods: Practice guidelines, systematic reviews, meta-analyses and randomized controlled trials (RCT) were searched using the keywords phimosis and steroids. The Strength of Recommendation Taxonomy (SORT) of the American Family Physician was used to classify the articles.

Results: Forty four articles were found and four met the inclusion criteria for this review. One guideline proposes treatment with a topical steroid, $0,05 \%$ to $0,1 \%$, twice a day for 20 to 30 days (SOR A). Three randomized controlled trials found that the use of topical steroids in the treatment of primary phimosis is effective, with a resolution rate of $65,8 \%$ to $90 \%$ and no reported adverse effects (evidence level 1).

Conclusions: The available evidence suggests that the use of topical steroids for the treatment of primary phimosis in pediatric patients is effective and without adverse effects (SOR A). The application of the drug requires retraction of the foreskin, which must be repeated for maintaining hygiene. Topical steroids are safe and effective for the treatment of phimosis and can be recommended by the Family Physician.

Keywords: Phimosis; Steroids. 